\title{
Using Branch Prediction Information for Near-Optimal I-Cache Leakage Reduction
}

University of Virginia, Department of Computer Science Tech Report CS-2006-03

\author{
Sung Woo Chung and Kevin Skadron \\ Department of Computer Science \\ University of Virginia \\ Charlottesville, VA 22904 \\ \{schung, skadron\}@cs.virginia.edu
}

\begin{abstract}
This paper describes a new on-demand wakeup prediction policy for instruction cache leakage control that achieves better leakage savings than prior policies, and avoids the performance overheads of prior policies. The proposed policy reduces leakage energy by more than $92 \%$ with only less than $0.3 \%$ performance overhead on average, whereas prior policies were either prone to severe performance overhead or failed to reduce the leakage energy as much. The key to this new on-demand policy is to use branch prediction information for the wakeup prediction. In the proposed policy, inserting an extra stage for wakeup between branch prediction and fetch, allows the branch predictor to be also used as a wakeup predictor without any additional hardware. Thus, the extra stage hides the wakeup penalty, not affecting branch prediction accuracy. Though extra pipeline stages typically add to branch misprediction penalty, in this case, the extra wakeup stage on the normal fetch path can be overlapped with misprediction recovery. With such consistently accurate wakeup prediction, all cache lines except the next expected cache line are in the leakage saving mode, minimizing leakage energy. We focus on super-drowsy leakage control using reduced supply voltage, because it is well suited to the instruction cache's criticality. The proposed policy can be applied to other leakage saving circuit techniques as long as the wakeup penalty is at most one cycle.
\end{abstract}

\section{Introduction}

Power dissipation has emerged as a major concern both for high-end processors and embedded processors, since higher power incurs higher packaging, power delivery and cooling costs. Recently, power dissipations have become high enough to cause serious thermal challenges, possibly even resulting in a project cancellation [26]. As process technology scales down, leakage energy accounts for a significant part of total energy. The International Technology Roadmap for Semiconductor [23] predicts that by the 70nm technology, leakage may constitute as much as $50 \%$ of total energy dissipation. In particular, the leakage energy for on-chip caches is crucial, since they comprise a large portion of chip area. For instance, 30\% of the Alpha 21264 and $60 \%$ of the StrongARM are devoted to cache and memory structures [13]. However, cache size can not be decreased to reduce leakage power since cache size is directly related to the performance.

There have been four major circuit techniques to reduce leakage energy dynamically: ABB (Adaptive-reverse Body Biasing) MTCMOS [17], DRG (Data-Retention Gated-ground) [1], Gated-Vdd [18], and DVS for Vdd (which is also called drowsy cache) [3]. In the ABB MTCMOS technique, threshold voltage is dynamically changed but the wakeup penalty between the active mode and the leakage saving mode is long, which makes it difficult for use in L1 caches [4]. DRG retains the data while reducing leakage by gating ground and using remaining leakage to retain cell contents, but the wakeup penalty is long. Thus, this technique may be inappropriate for timing critical caches such as an L1 cache, even if it is effective for less timing critical caches such as L2 [10]. The gated-Vdd technique reduces the leakage power by breaking the connection from the supply voltage (Vdd) or ground (the difference compared to DRG is that a larger sleep transistor is used and cell contents are not preserved) when the cell is put to sleep. While this technique dramatically reduces the leakage, its main disadvantage is that it does not preserve the state of the data in the sleep mode [4]. When the line is needed after it has been put into the leakage saving mode, the line must be refetched from a lower-level memory, which leads not only to additional dynamic energy consumption but also to performance degradation. To prevent these costs, conservative prediction policies should be employed [5][21][22]. Gated-Vdd may, however, be suitable for some L1 data caches where re-fetch penalty is short [12]. Another leakage saving technique is to lower the supply voltage. In this technique, data is not lost when the cache line is in the leakage saving mode (called "drowsy" mode). In the drowsy mode, data is retained, although it can not be accessed for read or write operation. Fortunately, most cache lines are unused for long periods due to temporal locality. Thus, by putting infrequently used cache lines into drowsy mode and keeping frequently accessed cache lines in the active mode, much leakage power is reduced without significant performance degradation. Please note that there is a wakeup penalty to restore the voltage level of the Vdd from the drowsy mode into the active mode. However, the wakeup penalty is expected to be one cycle in $70 \mathrm{~nm}$ process technology [3]. There has been concern that drowsy cache is more susceptible to soft errors than conventional caches [10]. Fortunately, instructions are read-only and 
must be protected by parity even in the absence of drowsy techniques. In the infrequent cases when an error is detected, the instruction only has to be refetched.

Among the above four techniques, drowsy technique is most suitable for L1 instruction caches, since it retains data and has short wakeup penalty. In order to prevent (or hide) the wakeup penalty of the drowsy cache, many prediction policies have been proposed. The easiest policy is "no prediction": to place all the cache lines into the drowsy mode periodically and restore the voltage level of Vdd of accessed cache lines, suffering the wakeup penalty. It performs well with data caches because they have high temporal locality, leading to little performance loss, and out-of-order processors can often tolerate extra latency from waking up lines [3]. For instruction caches, however, this "no prediction" technique does not perform well, because any wakeup penalty that stalls fetching directly impacts the performance. Many prediction policies have been proposed for instruction caches. (Details will be explained in the next section). None of them has simultaneously shown consistent leakage energy reduction with negligible performance degradation. In this paper, we propose a new on-demand wakeup prediction policy for an instruction cache. By on-demand, we mean that only the cache line currently in use needs to be awake. This technique takes advantage of the fact that we can accurately predict the next cache line by using the branch predictor. Thus, the wakeup prediction accuracy capitalizes on branch predictors that have already proven to be very accurate [14]. A further advantage compared to previous policies is that the proposed policy does not require an additional predictor. To utilize the branch predictor for wakeup prediction, we can allow a pipeline stage between branch prediction and instruction cache fetch. Allowing the branch predictor to be accessed one cycle earlier permits the branch prediction outcome to be used for wakeup, without harming branch prediction accuracy or requiring additional wakeup prediction hardware. Please note that this approach does not suffer the traditional branchmisprediction overhead of inserting extra stage in the pipeline. On a branch misprediction, the extra wakeup stage is overlapped with misprediction recovery. For further details, see Section 3.

This work focuses on use of drowsy cache (actually super-drowsy cache [9], explained in Section 2) as the leakage saving circuit technique, but if the wakeup penalty of the ABB MTCMOS or DRG, or the cache miss latency were shortened in the future, the on-demand policy works equally well with these leakage-saving circuit techniques. In this case, the proposed policy can be also applied to the leakage saving circuit techniques. In this paper, we distinguish the wakeup prediction policy from the leakage saving circuit technique. The wakeup prediction policy predicts which cache line will be woken up, while the leakage saving circuit technique is the mechanism for putting lines to sleep and waking them up, independent of the prediction policy.

The rest of this paper is organized as follows. Section 2 explains the concept of the drowsy/super-drowsy cache and presents previously proposed prediction policies for a drowsy instruction cache. Section 3 proposes a new ondemand wakeup prediction policy by using branch prediction information. Section 4 presents the analytical model for evaluation and simulation environments. Section 5 evaluates energy/performance for the proposed policy. Finally we provide conclusions in Section 6.

\section{Background Work}

\subsection{Drowsy/Super-Drowsy Cache Circuit Technique}

The drowsy cache technique [3] has received a great deal of attention, because it retains data while providing a short wakeup penalty. When the cache line is not expected to be used in the near future, the Vdd of the cache line is reduced to a lower level, leading to lower leakage power. In active mode when normal voltage is supplied, the cache line operates the same as in a conventional cache. In drowsy mode, however, the cache line cannot be accessed even though data is retained. After being woken up, the cache line is accessed. Since the drowsy mode does not fully turn off the supply voltage, the drowsy cache does not reduce the leakage power as much as gated-Vdd, but data retention means the drowsy cache does not need to refetch instructions. Moreover, wakeup penalty is short: one cycle is expected in $70 \mathrm{~nm}$ technology [3].

Kim et.al proposed a refinement of this technique, called super-drowsy cache [9]. A single-Vdd cache line voltage controller with Schmitt trigger inverter replaces multiple supply voltage sources in order to alleviate interconnect routing space. In addition, the on-demand gated bitline precharge technique [20] is employed to reduce the bitline leakage. We apply our prediction policy to the super-drowsy cache because it is the most advanced circuit technique for instruction cache leakage control as far as we know.

\subsection{Previous Wakeup Prediction Policies}

The success of the drowsy-style cache depends on how accurately the next cache line can be predicted and woken up. Especially for an instruction cache, accuracy is crucial since the accuracy directly affects performance degradation. A simple policy is noaccess [3]: This uses per-line access history and puts all the unused lines into drowsy mode periodically. For more accurate wakeup prediction, two prediction policies were proposed for a 
drowsy instruction cache [8] - NSPB (Next Subcache Prediction Buffer) and NSPCT (Next Subcache Predictor in Cache Tags). Additional storage is required to predict the next subbank (not a cache line) using NSPB, whereas cache tags are extended to provide the subbank predictor in NSPCT. Therefore, NSPCT requires less hardware overhead but is comparable to NSPB in accuracy (performance loss is $0.79 \%$ ). However, leakage reduction is weak [8] due to large sub-bank turn-on energy. Zhang et.al. proposed the Loop policy [22] where all cache lines are put into the drowsy mode after each loop was executed. This bears some similarity to the DHS (Dynamic HotSpot Based Leakage Management) policy, which was proposed in [5]. DHS makes use of the branch target buffer (BTB), since branch behavior is an important factor in shaping the instruction access behavior. In the DHS policy, the global turnoff (drowsy) signal is issued when a new loop-based hotspot is detected. Thus this policy can lower the supply voltage of unused cache lines before the update window expires by detecting that execution will remain in a new loop-based hotspot. The DHS-PA (DHS-Per Access) policy employs a Just-In-Time-Activation (JITA) strategy on top of the DHS policy [5]. The JITA strategy is to wake up the next sequential line, exploiting the sequential nature of code. However, this is not successful when a taken branch is encountered. The DHS-Bank-PA policy [5] issues the global turn-off signal at fixed periods, when the execution shifts to a new bank, or when a new loop hotspot is detected. It attempts to identify both spatial and temporal locality changes. It also employs hotspot detection to protect active cache lines and the JITA policy for predictive cache line wakeup. As shown in [5], although the DHSBank-PA reduced leakage energy significantly, performance degradation is severe.

The super-drowsy cache deploys the noaccess-JITA policy with as large as a 32K-cycle update window size for next cache line prediction to achieve high accuracy [9]. The noaccess-JITA puts only lines that have not been accessed during a fixed time period into drowsy mode and activates the first sequential cache line. The super-drowsy cache also deploys an additional NTSBP (Next Target Sub-Bank Predictor) that predicts next sub-bank to be bitline precharged in advance, since the on-demand gated precharge incurs extra penalty to enable an inactive sub-bank, and this can result in significant execution time increase. The noaccess-JITA/NTSBP with 32K cycle update window size is a leakage energy reduction policy with the most accurate wakeup prediction but with modest leakage energy reduction. However, the accuracy of the noaccess-JITA/NTSBP is so dependent on program behavior, especially locality, that the accuracy of no-access-JITA/NTSBP is poor in some applications.

For our study, we selected two policies: noaccess-JITA/NTSBP and DHS-Bank-PA, since the former is known to be the most accurate and the latter is known to reduce leakage reduction most. For a fair comparison, we also apply the gated bitline precharging technique to all the policies. Note that there is no additional penalty for on-demand bitline precharging in DHS-Bank-PA, where all the cache lines in the previous sub-bank are put into the drowsy mode when execution moves to a new sub-bank. All these previously proposed policies are explained in Table 1 to prevent possible confusion.

\begin{tabular}{|l|l|}
\hline \multicolumn{1}{|c|}{ Policy } & \multicolumn{1}{c|}{ Description } \\
\hline \hline Noaccess & $\begin{array}{l}\text { All the unused cache line is put into drowsy mode } \\
\text { periodically by referencing to per-line access history. }\end{array}$ \\
\hline NSPB (Next Subcache Prediction Buffer) & Additional predictor to wake up the next subbank \\
\hline NSPCT (Next Subcache Predictor in Cache Tags) & Additional bits in cache tags to wake up the next subbank \\
\hline NTSBP (Next Target Sub-Bank Predictor) & Additional predictor to precharge the bitline of a sub-bank \\
\hline Loop & $\begin{array}{l}\text { All cache lines are put into the drowsy mode after each } \\
\text { loop was executed }\end{array}$ \\
\hline DHS (Dynamic HotSpot based leakage management) & $\begin{array}{l}\text { All cache lines are put into the drowsy mode when a new } \\
\text { loop-based hotspot is detected }\end{array}$ \\
\hline DHS-PA (Per Access) & In addition to DHS, next cache line activation is supported \\
\hline Noaccess-JITA (Just In Time Activation)/NTSBP & $\begin{array}{l}\text { For a drowsy cache, Noaccess is used, while activating } \\
\text { sequentially next cache line. For bitline gating, NTSBP is } \\
\text { used }\end{array}$ \\
\hline DHS-Bank-PA & $\begin{array}{l}\text { For a drowsy cache, DHS-Bank is used while activating } \\
\text { sequentially next cache line. Automatically DHS-Bank-PA } \\
\text { predicts next sub-bank to be bitline precharged }\end{array}$ \\
\hline
\end{tabular}

Table 1. Previously proposed prediction policies, including the policies for comparison

\section{Novel Wakeup Prediction Policy : Utilizing Branch Prediction Information}

In conventional drowsy (including super-drowsy) instruction caches, branch predictors are only used to predict the branch direction/target. In previous wakeup prediction policies, additional predictors are required in order to 
wake up a cache line, and accessed cache lines remain active for a fixed time period. Accordingly, the accuracy of the previous policies is highly dependent on the locality. As shown in Figure 1(a), the additional predictors, such as JITA [5], NSPB [8], NSPCT [8] and NTSBP [9], are accessed before looking up the branch predictor in order to hide the wakeup penalty. However, the accuracy of additional predictors was not satisfactory. For near-optimal leakage energy reduction and performance, we propose a new wakeup prediction policy which enables on-demand wakeup. In the proposed policy, as shown in Figure 1(b), the branch predictor, consisting of Prediction History Table (PHT) and Branch Target Buffer (BTB), is accessed one cycle earlier than in conventional policies.

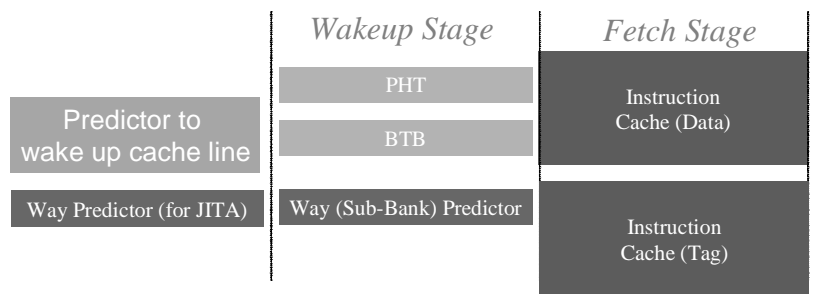

(a) Conventional Drowsy Instruction Cache

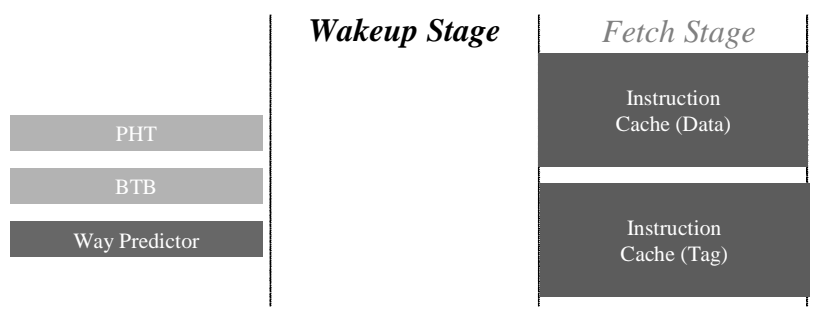

(b) Proposed Drowsy Instruction Cache

Figure 1. Pipeline stage comparison

There are two architectural options in branch resolution. When a branch turns out to be mispredicted in the execution stage, some time is usually required to clean up mis-speculated state and generate the next address (Figure 2(a)), but depending on exactly where during the branch-resolution cycle the misprediction is detected, it may be possible to complete this without any extra overhead (Figure 3(a)). Requiring at least one cycle for cleanup and fetch-address generation appears to be common [23].

- Additional penalty for recovery after the execution stage

As shown in Figure 2 (b), after the execution/branch-resolution stage of the instruction $n$, cleanup, effective address calculation, and wakeup occur simultaneously. Thus there is always only one active cache line.

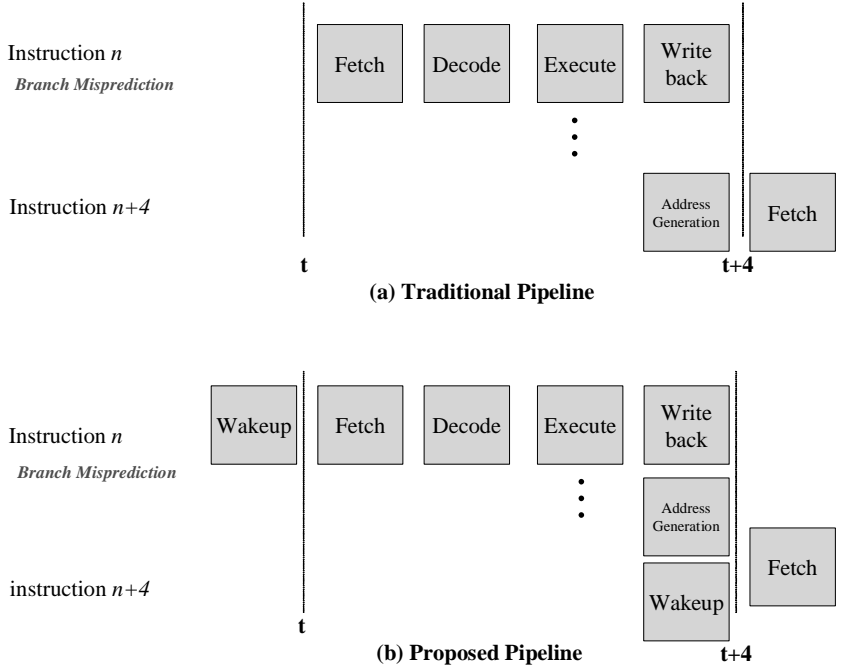

Figure 2. Pipeline structure (when there is one-cycle penalty for effective address calculation) 


\section{- No penalty for recovery after the execution stage}

In Figure 3 (b), it is impossible to wake up only the one correct cache line after a misprediction without incurring a one-stage penalty, because cleanup and address generation occur in the same stage as misprediction detection. Instead, the potential alternative path should be woken up speculatively in parallel with branch resolution. This means that during some cycles, two lines are awake.
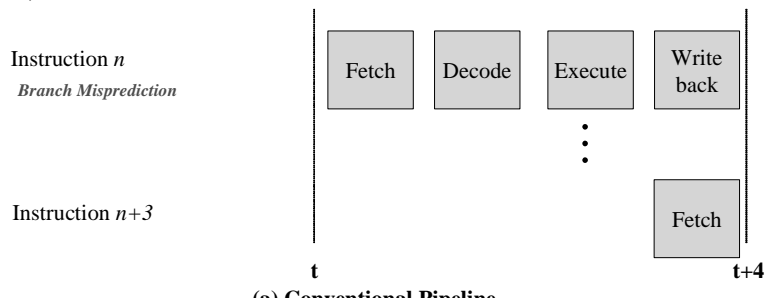

(a) Conventional Pipeline

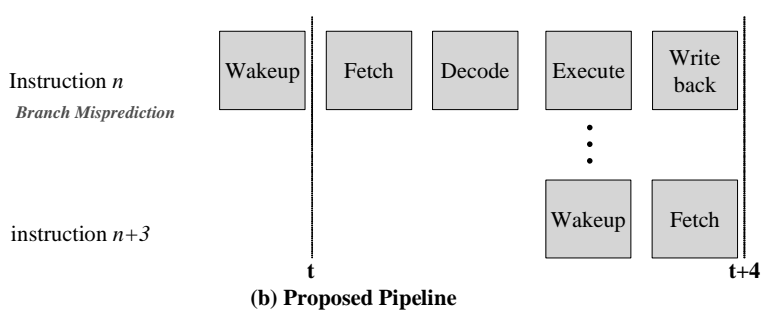

Figure 3. Pipeline structure (when there is no penalty for effective address calculation)

It is possible to determine the alternative path in parallel with branch resolution. For predicted-taken branches, the not-taken path must be woken up and the branch address itself (usually carried with the instruction) can be used. For predicted not-taken branches, the taken target is needed. This can either be carried with the instruction or reside in some dedicated storage. This capability must exist anyway in current microprocessors because every taken branch in flight must be able to check whether the target address obtained from the BTB is correct or not. Note that the taken target is available at branch-prediction time regardless of predicted direction, because the direction predictor and target predictor are usually consulted in parallel.

There are only two cases that we can not cover in case of branch direction misprediction. Since the cache line address woken up is not that of (mispredicted instruction address +4$)$, but the mispredicted instruction address itself, there is a penalty when the mispredicted instruction is at the end of the cache line. It is possible to make use of the instruction address +4 , but it requires extra adder or storage for the instruction address +4 . Even though this cost may be minor, in this paper we do not use an extra adder or extra storage, since the probability that a mispredicted instruction is at the end of the cache line is rare.

In the proposed policy, only one cache line (or two cache lines in Figure 3) expected to be accessed exists in the active mode and all the other cache lines are in the drowsy mode. After a new line is selected for wakeup, the current awake line is put to sleep. For a set-associative cache, only one way should woken up to save the energy. We adopt a way predictor [19] that employs MRU (Most Recently Used) bit and integrates a way predictor and a BTB for high accuracy, which is known as one of the most accurate way predictors. For conventional drowsy instruction caches, the way predictor is used to predict the way that will be woken up. Conventional way predictors only wake up the cache line that is sequential. Non-sequential cache lines are expected to still be awake based on cache lines that were previously woken up and remain active. In the noaccess-JITA/NTSBP, the way predictor is used for cache line wakeup prediction, while for NTSBP it is used for precharging and way prediction of cache line to be read. When the way predictor can have 2-read ports in order to predict the next cache line that will be actually read as well, the prediction accuracy for precharging is higher and the NTSBP is unnecessary (In this paper, we call this policy as Noaccess-JITA utilizing w.p. (Way Predictor)). Both options (noaccess-JITA/NTSBP and noaccess-JITA (utilizing w.p.) are evaluated in this paper. In DHS-Bank-PA, way prediction is not required in case of actual cache read, since the whole sub-bank is put in the sleep mode when execution jumps from one sub-bank to another, resulting in overlapping of wakeup penalty and precharging penalty. In the proposed policy, the PHT and the BTB are accessed one cycle earlier, which leads to one cycle earlier way prediction. There is no need for another way prediction to read the instruction cache, since only one woken up cache line can be read in the proposed on-demand policy. In case of Figure 3, however, a two-port way predictor is required to support concurrent two accesses: one is to wake 
up the next cache line in case of correct branch prediction (to wake up instruction $n+3$, when instruction $n$ is predicted correctly in Figure 3 (b)) and the other is to wake up a probable cache line recovered from branch misprediction (to wake up instruction $n+3$, when instruction $n$ is recovered from branch misprediction in Figure 3 (b)).

Figure 4 shows one example of the proposed policy. After a misprediction by incorrect target address, the recovered target address $(0 \mathrm{x} 00182 \mathrm{f} 10)$ is woken up in cycle $\mathrm{n}$. At the same time, the branch predictor is looked up to predict next fetch block. In the conventional pipeline, the first branch predictor access is done in cycle $n+1$. There is no predicted taken branch in the fetch block (0x00182f10), leading to waking up the next sequential fetch block $(0 \mathrm{x} 00182 \mathrm{f} 20)$ in cycle $\mathrm{n}+1$. In cycle $\mathrm{n}+1$, the branch predictor is accessed for the block $(0 \mathrm{x} 00182 \mathrm{f} 20)$, which should be accessed in cycle $n+2$ in the conventional pipeline. In this case, the fetch block (0x00182f20) has a predicted taken branch. Thus, the target address from the BTB is used for wakeup address. Accordingly, the block (0x001820a0) is woken up in cycle $n+2$ and fetched in cycle $n+3$. Please note that the proposed policy does not affect the branch prediction accuracy.

Since the non-branch instruction accounts for large portions of instructions and the branch prediction accuracy is high, the proposed policy is expected to be accurate, with the advantage of maximum leakage reduction.

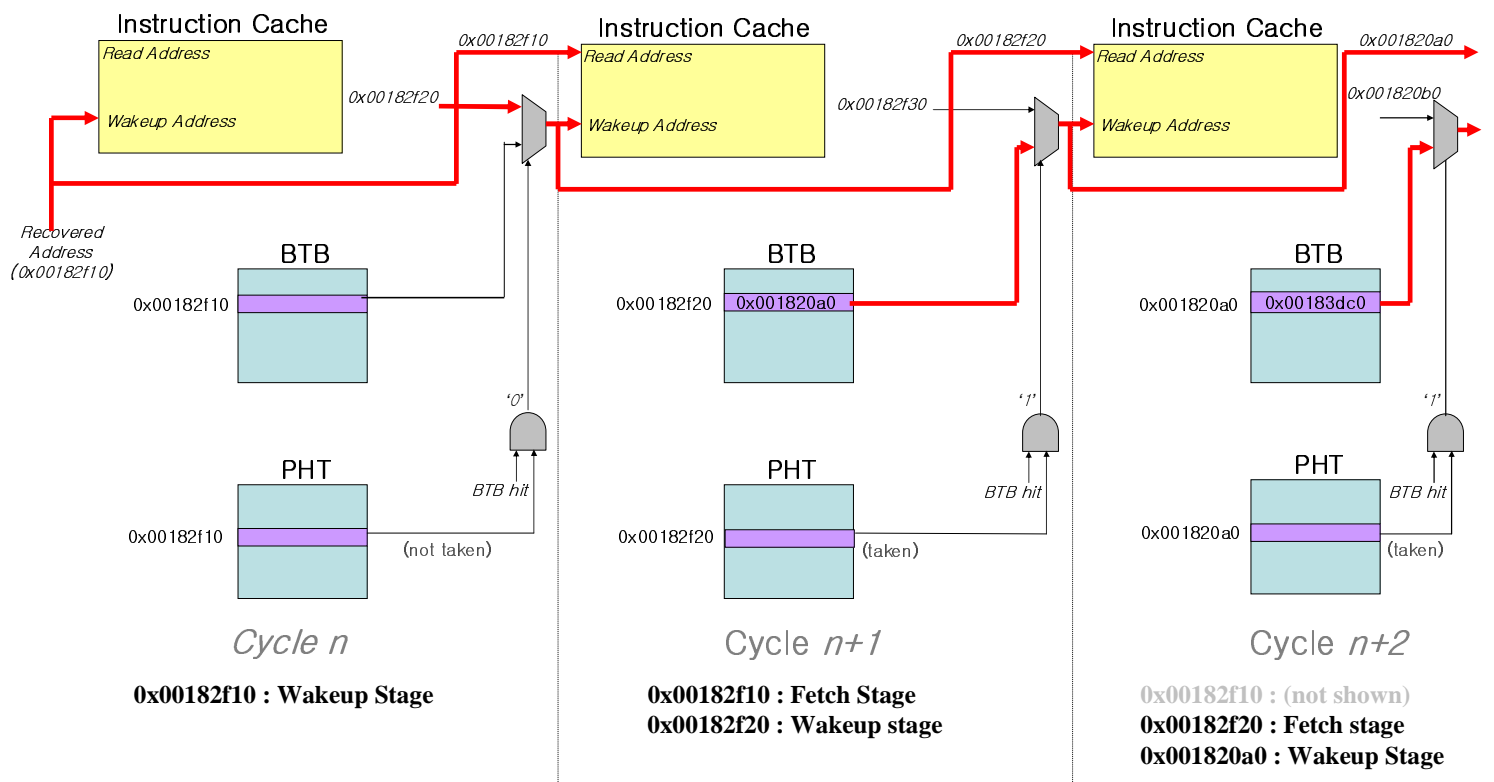

Figure 4. Example operation of the on-demand wakeup prediction policy, in which fetch resumes at address 0x0018sf10 after a misprediction.

\section{Experimental Methodology}

\subsection{Analytical Models}

Leakage-Related Energy includes leakage energy in the active mode, leakage energy in the drowsy mode (including super-drowsy mode), turn-on energy for prediction, and turn-on energy for correction of wakeup misprediction (turn-on means changing the cache line from the drowsy mode into the active mode and is due to the need to charge the capacitance of the line back to Vdd). The leakage energy due to extra execution time is captured, since the increased total execution time affects leakage-related energy in formula (1).

\begin{tabular}{|l|l|}
\hline Notation & Description \\
\hline \hline $\mathrm{P}_{i}$ & Leakage power consumed for a cache line in the $i^{\text {th }}$ mode \\
\hline $\mathrm{P}_{\text {turn-on }}$ & Transition power consumed for a cache line to be changed to the active mode \\
\hline $\mathrm{AN}_{i}$ & Average Number of cache lines in the $i$ mode \\
\hline $\mathrm{TN}_{\text {prediction }}$ & Total Number of cache lines turned on by wakeup prediction \\
\hline $\mathrm{TN}_{\text {correction }}$ & Total Number of cache lines turned on for correction (by wakeup misprediction) \\
\hline $\mathrm{T}$ & Total execution time \\
\hline
\end{tabular}

Table 2. Notation description 
Leakage-Related energy is calculated as follows. The notations are described in Table 2.

$$
\mathrm{E}_{\text {leakage_related }}=\sum \mathrm{P}_{i} * \mathrm{AN}_{i} * \mathrm{~T}+\mathrm{P}_{\text {turn-on }} *\left(\mathrm{TN}_{\text {prediction }}+\mathrm{TN}_{\text {correction }}\right)
$$

where $i=$ active mode, drowsy mode, bitline gating mode, super-drowsy mode (drowsy mode + bitline gating mode) $\}$

In the base model, all the cache lines are always in the active mode. The policies use predictions to reduce the leakage-related energy by decreasing the number of cache lines in the active mode. In the proposed policy, all the cache lines except the next fetch cache line are in the drowsy mode, in which some of them are bitline-gated and others are not.

In order to compare the proposed policy to the theoretically best policy, the optimal policy is presented in this paper. The optimal policy is assumed to have perfect knowledge of the future address trace. Thus, its performance is same as the base model and its leakage-related energy is least. Not only minimizing the number of cache lines in the active mode but also reducing the unnecessary turn-on energy is the goal of the optimal policy. If the turn-on energy is more than the active leakage energy (in other words, if the time until the cache line is reused is short enough), it is more efficient to leave the cache line in the active mode, instead of putting it in the drowsy mode. Accordingly, the optimal policy can save more energy than the proposed policy. Using the following formula (2), extended from [15], the optimal point can be found. If the reuse interval $\left(\mathrm{I}_{\text {reuse }}\right)$ for a cache line satisfies the following formula, it is more energy efficient to leave the cache line in the active mode.

$$
\mathrm{I}_{\text {reuse }} * \mathrm{P}_{\text {active_mode }}<\mathrm{I}_{\text {reuse }} * \mathrm{P}_{\text {drowsy_mode }}+\mathrm{P}_{\text {turn-on }}
$$

\subsection{Simulation Environment}

We extended Simplescalar 3.0 [2] to evaluate energy and performance. The processor parameters model a highperformance microprocessor similar to Alpha 21264 [7], as shown in Table 3. Table 3 also gives the technology and power/energy parameters used in this paper. The power/energy parameters are based on the $70 \mathrm{~nm} / 1.0 \mathrm{~V}$ technology [9]. We use all integer and floating point applications from the SPEC2000 benchmark suite [25] and use their alpha binaries and reference inputs for execution. Each benchmark is first fast-forwarded half a billion instructions and then simulated the next half a billion instructions.

We selected gshare for a branch predictor, since gshare performs fairly well and it is suspected to be used for Intel P4 architecture [16]. However, if more accurate branch predictor were selected for evaluation, the proposed policy would perform even better, while it would not help wakeup prediction accuracy for other policies. Using gshare, instead of a more aggressive branch predictor, therefore sets a higher bar for our proposed policy.

We selected three previous prediction policies (noaccess-JITA/NTSBP, noaccess-JITA (utilizing w.p.), and DHSBank-PA, described in Section 2 and Section 3) for comparison. We use same details of the policies as proposed in [5][9]. The noaccess-JITA/NTSBP has a $32 \mathrm{~K}$ cycle update window to periodically update mode of each cache line. Although execution moves from one sub-bank to another sub-bank, the precharge circuits of the previous sub-bank remain on for 16 cycles to prevent the misprediction of sub-bank. After 16 cycles, the bitline of the sub-bank is isolated. The DHS-Bank-PA has $2 \mathrm{~K}$ cycle update window and its hotness threshold (when the access count exceeds over this value, the cache line is considered to be a hotspot and all other cache lines except this hotspot basic block are turned off) is 16 .

\begin{tabular}{|l||l|}
\hline \multicolumn{2}{|c|}{ Processor Parameters } \\
\hline Instruction Window & 64 RUU, 32 LSQ \\
\hline Fetch/Decode/Issue/Commit Width & 4 instructions/cycle \\
\hline Branch Predictor & Gshare/4K, 1024-entry 4-way BTB \\
\hline Integer ALUs/Multi-divs/memory ports & $4 / 1 / 2$ \\
\hline FP ALUS/multi-divs & $4 / 1$ \\
\hline FU Latencies & $\begin{array}{l}\text { Int : mul 3, div 20, all others 1 } \\
\text { FP : adder 2, mul 4, div 12, sqrt 24 }\end{array}$ \\
\hline Memory Bus Width/Latency & 4 Bytes/80 and 8 cycles for the first and inter chunks \\
\hline Instruction/Data TLB & $\begin{array}{l}128 \text { entry/32 entry in each way, 8KB page size, fully } \\
\text { associative, LRU, 28-cycle latency }\end{array}$ \\
\hline L1 I-Cache & $\begin{array}{l}32 \mathrm{~KB}, 1 \text { way/4 way, 32B blocks, 1 cycle latency, } \\
\text { 4KB sub-bank size }\end{array}$ \\
\hline L1 D-Cache & $32 \mathrm{~KB}, 4$ ways, 32B blocks, 1 cycle latency \\
\hline L2 Unified Cache & 512 KB, 4 ways, 64B blocks, LRU, 12 cycle latency \\
\hline
\end{tabular}




\begin{tabular}{|l||l|}
\hline \multicolumn{2}{|c|}{ Power/Energy Parameters } \\
\hline Process Technology & $70 \mathrm{~nm}$ \\
\hline Threshold Voltage & $0.2 \mathrm{~V}$ \\
\hline Supply Voltage & $1.0 \mathrm{~V}$ (active mode), 0.25 V (drowsy mode) \\
\hline $\begin{array}{l}\text { Leakage Power/Bit in Active Mode w/o Gated } \\
\text { Precharging (1 cycle) }\end{array}$ & $0.0778 \mu \mathrm{W}$ \\
\hline $\begin{array}{l}\text { Leakage Power/Bit in Active Mode w/ Gated } \\
\text { Precharging (1 cycle) }\end{array}$ & $0.0647 \mu \mathrm{W}$ \\
\hline $\begin{array}{l}\text { Leakage Power/Bit in Drowsy Mode w/o Gated } \\
\text { Precharging (1 cycle) }\end{array}$ & $0.0167 \mu \mathrm{W}$ \\
\hline $\begin{array}{l}\text { Leakage Power/Bit in Drowsy Mode w/ Gated } \\
\text { Precharging (1 cycle) }\end{array}$ & $0.00387 \mu \mathrm{W}$ \\
\hline Turn-on (drowsy to active) Energy/Bit & $115 \mathrm{fJ}$ \\
\hline Turn-on (drowsy to active) Latency & 1 cycle \\
\hline Clock Cycle Time & $12 * \mathrm{FO} 4(395 \mathrm{ps})$ \\
\hline
\end{tabular}

Table 3. Architecture/circuit parameters

\section{Simulation Results}

This section presents our simulation results and compares the proposed policy to other policies. We analyze each policy's energy reduction and execution time increases. Then, we explore the effects of the proposed policy on the total processor energy and its potential effect on other leakage saving circuit techniques.

\subsection{Drowsy Fraction and Gated Bitline Precharging Fraction}

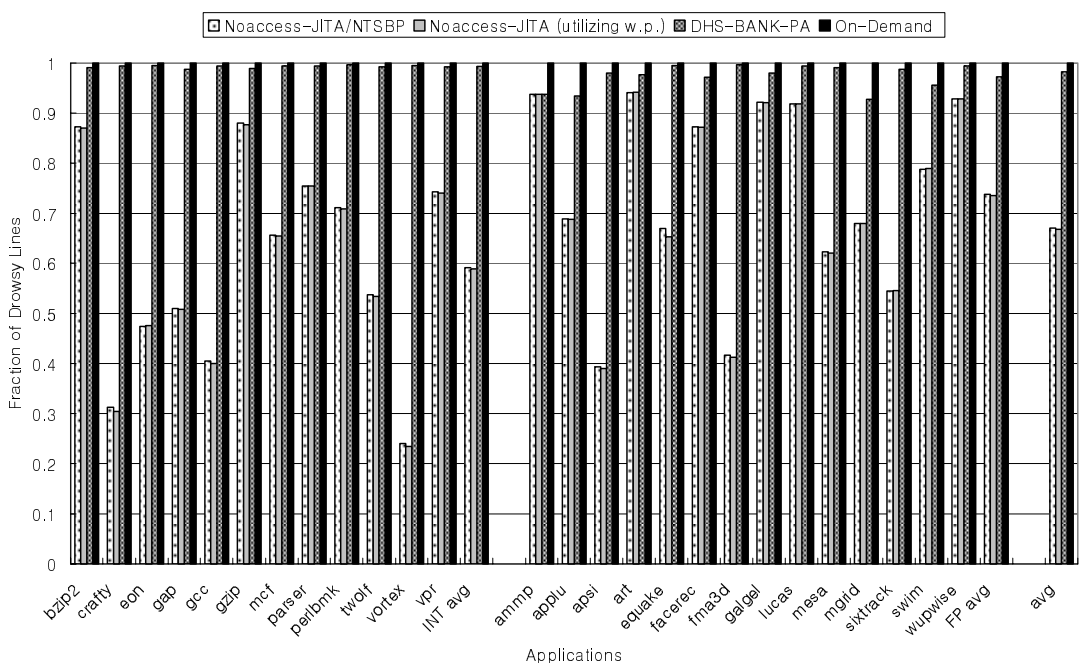

Figure 5. Average drowsy fraction in instruction cache (4-way set-associative)

Figure 5 shows the drowsy fraction in the 4-way set-associative cache. We do not show the drowsy fraction in the direct-mapped cache, because the results are similar to the 4-way set associative cache. Since the update window size of the noaccess-JITA/NTSBP is as large as 32K, the drowsy fraction is $66.9 \%$ (65.3\% in the direct-mapped cache), on average. In the DHS-Bank-PA, the drowsy fraction is $98.2 \%$ (97.8\% in the direct-mapped cache), on average. The reason is that the update window size is as small as $2 \mathrm{~K}$ and additionally cache lines are put into the drowsy mode when a new hotspot is detected. In the proposed on-demand policy, only one (or two in the proposed policy of Figure 3) cache line is in the active mode and the others are in the drowsy mode, resulting in $99.9 \%$ (or $99.8 \%$ in the proposed policy of Figure 3) drowsy fraction, on average. Naturally, the direct-mapped cache has also $99.9 \%$ (or $99.8 \%$ in the proposed policy of Figure 3) drowsy fraction, on average. There is little difference between the noaccess-JITA/NTSBP and the noaccess-JITA (utilizing w.p.), since the NTSBP and the 2-read port way predictor are not related to the drowsy fraction but related to the precharging fraction. We only show the on-demand policy of Figure 2 instead of that of Figure 3, since there is only negligible difference $(0.1 \%)$ between them.

Figure 6 shows the fraction of isolated bitines in the 4-way set associative cache. We do not show the fraction of 
isolated bitlines in the direct-mapped cache, since the results are similar to the 4-way set associative cache. In case of bitline precharging prediction, there is no energy penalty but there is one cycle timing penalty when mispredicted. In the noaccess-JITA/NTSBP, on average $75.7 \%$ (77.1\% in the direct-mapped cache) of the sub-banks are bitline gated. The fraction is relatively small, because a sub-bank should be remained bitline precharged for 16 cycles to prevent bitline precharging mispredictions when execution moves to another sub-bank. However, the noaccess-JITA (utilizing w.p.) always has $87.5 \%$ since way predictor is used for subbank prediction. In the other two techniques, only one sub-bank is bitline percharged. Thus, the portion of gated bitline precharging is always $87.5 \%$ ( 1 subbank/8 sub-banks). We only show the on-demand policy of Figure 2, since there is negligible difference between them.

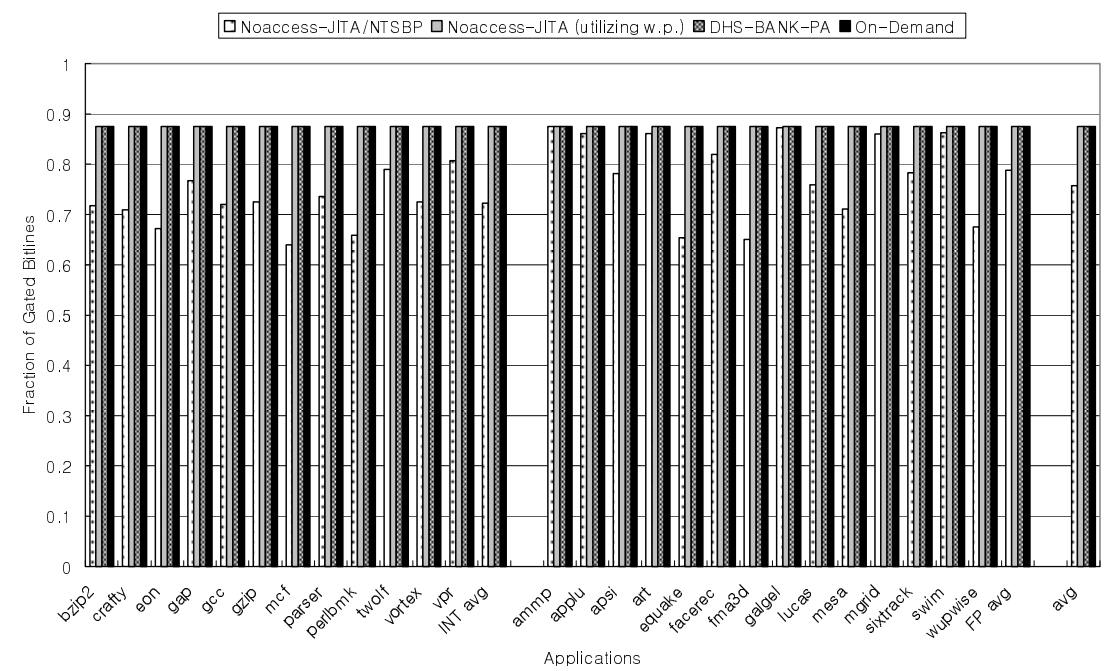

Figure 6. Average isolated bitline fraction in instruction cache (4-way set-associative)

\subsection{Total Leakage-Related Energy}

Figure 7 shows normalized leakage-related energy to the base model in the direct-mapped cache (As explained in Section 4.1, the base model is a conventional cache that does not perform leakage control). The noaccessJITA/NTSBP shows inconsistent reductions depending on applications, whereas the other policies show very consistent reductions. Average leakage-related energy reduction is $67.5 \%, 91.3 \%, 92.5 \%, 92.2 \%$, and $92.6 \%$ in the noaccess-JITA/NTSBP, DHS-Bank-PA, on-demand of Figure 2, on-demand of Figure 3, and optimal policies, respectively.

In the proposed policy, the next cache line is woken up on-demand. Thus, the leakage energy in the active mode is minimized, whereas turn-on energy by prediction is expected to be larger due to more frequent sleep/activation round-trips compared to the other previous policies, such as the noaccess-JITA/NTSBP and the DHS-Bank-PA. However, turn-on energy in the proposed policy still accounts for a small portion of total leakage-related energy. Consequently, the average relative difference in leakage-related energy between the proposed policy and the optimal policy is only $1.6 \%$ (6.4\% for the proposed policy of Figure 3). In contrast, the leakage-related energy difference between the DHS-Bank-PA and the optimal policy is $18.4 \%$. The proposed policy of Figure 3 consumes a little more energy than that of Figure 2, since there is unnecessary turn-on energy. 


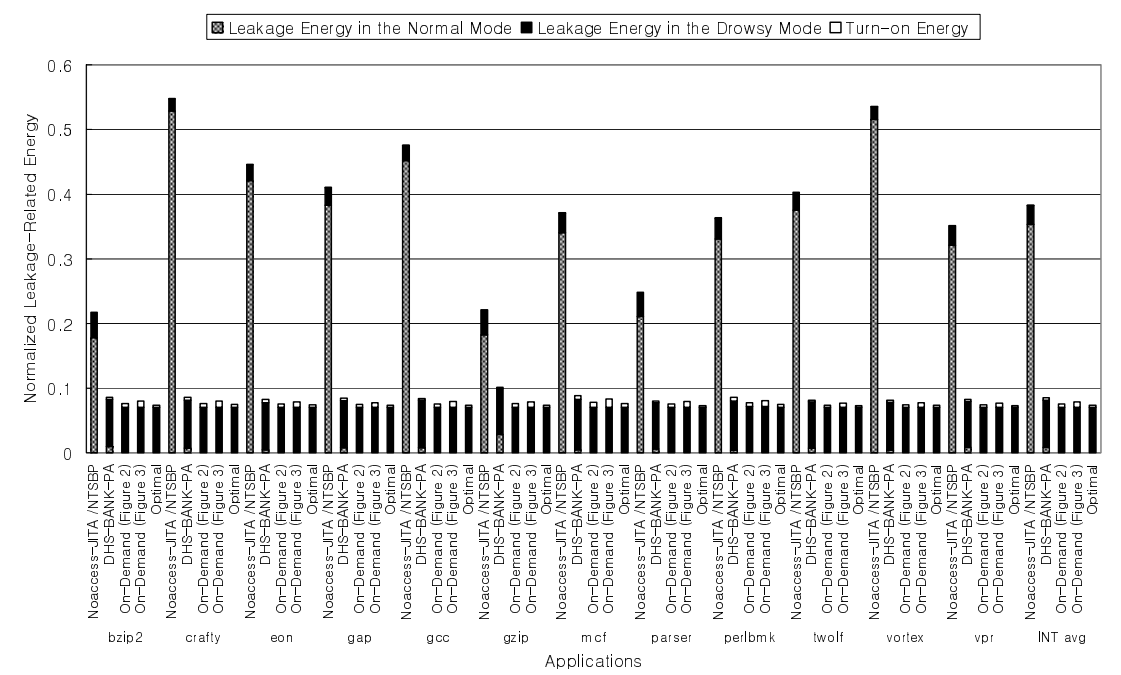

(a) SPEC2000 INT applications

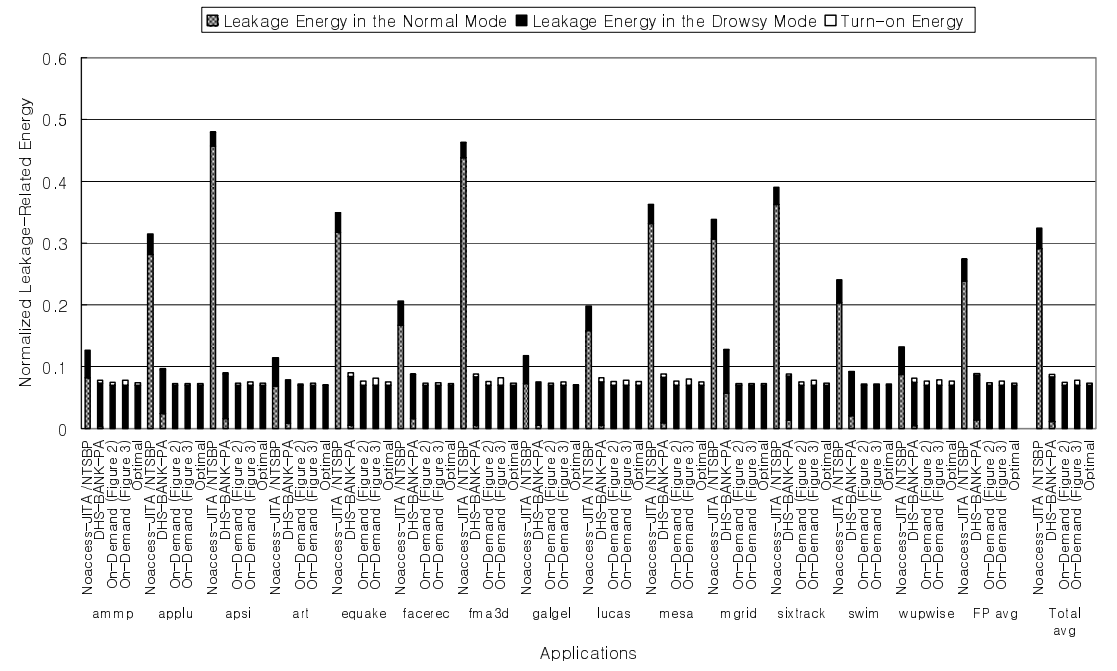

(b) SPEC2000 FP applications and total average

Figure 7. Normalized leakage-related energy in the direct-mapped cache

Figure 8 shows normalized leakage-related energy to the base model in the 4-way set-associative cache. The base model does not use any leakage-saving policy but it has the way predictor. Average leakage-related energy reduction is $68.1 \%, 69.8 \%, 90.4 \%, 92.5 \%, 92.2 \%$, and $92.6 \%$ in the noaccess-JITA/NTSBP, noaccess-JITA (utilizing w.p.), DHS-Bank-PA, on-demand of Figure 2, on-demand of Figure 3, and optimal policies, respectively. These results are similar to the results in the direct-mapped cache. 
Leakage Energy in the Normal Mode Leakage Energy in the Drowsy Mode QTurn-on Energy

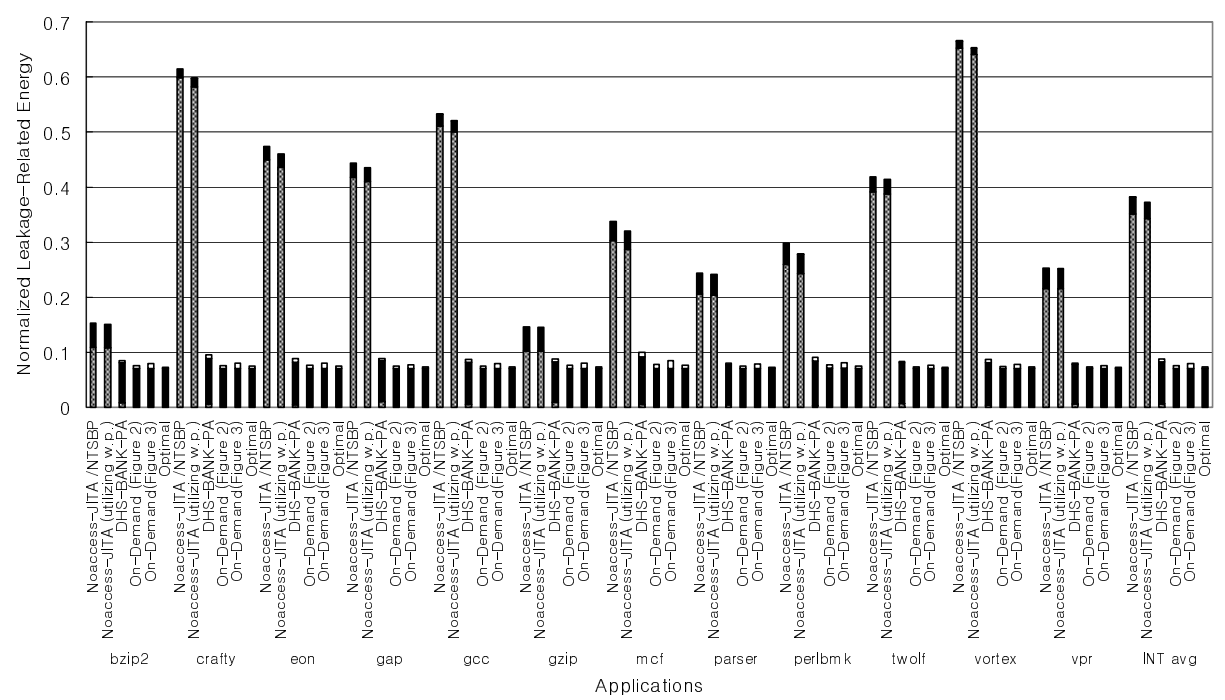

(a) SPEC2000 INT applications

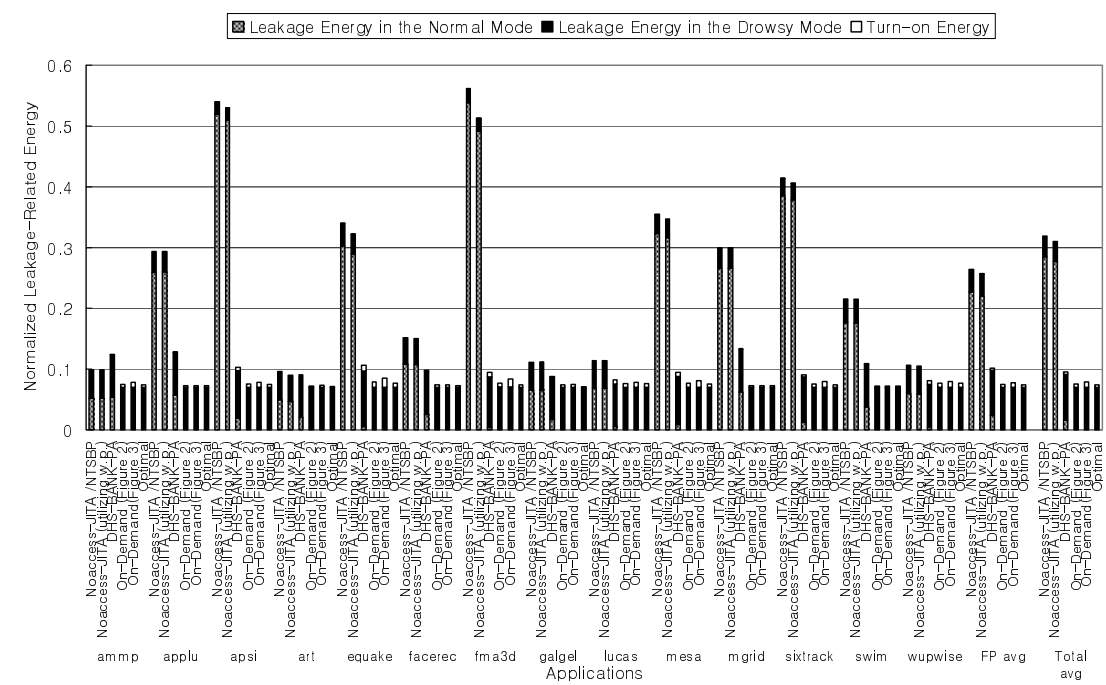

(b) SPEC2000 FP applications and total average

Figure 8. Normalized leakage-related energy in the 4-way set-associative cache

\subsection{Wakeup Prediction Accuracy}

Table 4 shows the branch prediction accuracy and the branch instruction ratio (\#branch instructions/\#total instructions) for SPEC2000 applications. On average, the branch prediction accuracy is $94.3 \%$ and the branch instruction ratio is $8.7 \%$. Recall that wakeup misprediction is mainly caused by branch misprediction by incorrect target address. As the number of branch instructions gets smaller, the branch prediction accuracy affects wakeup prediction accuracy less. For example, gcc and gzip shows similar branch prediction accuracy but the branch instruction ratio of gzip is much less than that of gec, resulting in higher wakeup prediction accuracy of gzip in Figure 9 and Figure 10. 


\begin{tabular}{|l|r||l|r|}
\hline \multicolumn{1}{|c|}{$\begin{array}{c}\text { INT } \\
\text { Application }\end{array}$} & $\begin{array}{c}\text { Branch Prediction Accuracy (\%) } \\
\text { (Branch Instruction Ratio (\%) }\end{array}$ & $\begin{array}{c}\text { FP } \\
\text { Application }\end{array}$ & $\begin{array}{c}\text { Branch Prediction Accuracy (\%) } \\
\text { (Branch Instruction Ratio (\%)) }\end{array}$ \\
\hline bzip2 & $93.75(12.18)$ & ammp & $100.00(13.78)$ \\
\hline crafty & $88.73(11.48)$ & applu & $98.47(0.24)$ \\
\hline eon & $93.78(11.35)$ & apsi & $96.74(6.71)$ \\
\hline gap & $92.66(6.42)$ & art & $96.59(11.32)$ \\
\hline gcc & $89.43(16.06)$ & equake & $98.78(16.88)$ \\
\hline gzip & $90.16(9.36)$ & facerec & $98.72(3.97)$ \\
\hline mcf & $97.85(17.60)$ & fma3d & $96.25(8.09)$ \\
\hline parser & $94.15(15.70)$ & galgel & $98.91(5.78)$ \\
\hline perlbmk & $79.25(13.42)$ & lucas & $100.00(4.76)$ \\
\hline twolf & $91.47(14.36)$ & mesa & $95.90(10.85)$ \\
\hline vortex & $95.48(16.03)$ & mgrid & $95.46(0.31)$ \\
\hline vpr & $86.53(11.07)$ & sixtrack & $93.84(12.21)$ \\
\hline & & swim & $99.63(1.65)$ \\
\hline & & wupwise & $100.00(4.08)$ \\
\hline \hline INT avg & $90.27(12.92)$ & FP avg & $97.80(7.90)$ \\
\hline \hline & & Total Avg & $94.32(8.68)$ \\
\hline
\end{tabular}

Table 4. Branch prediction accuracy and branch instruction ratio

As explained in Section 2.2, correct cache line prediction for drowsy cache does not always mean correct subbank prediction for bitline precharging in the noaccess-JITA/NTSBP, since the cache line is predicted by noaccessJITA and the sub-bank is predicted by NTSBP (In other words, cache lines in the active mode are spread across subbanks). The same is applied to the noaccess-JITA (utilizing w.p.) in the set-associative cache. In the other policies, cache lines in the active mode are in one sub-bank.

口Noaccess-JITA/NTSBP ODHS-BANK-PA On-Demand

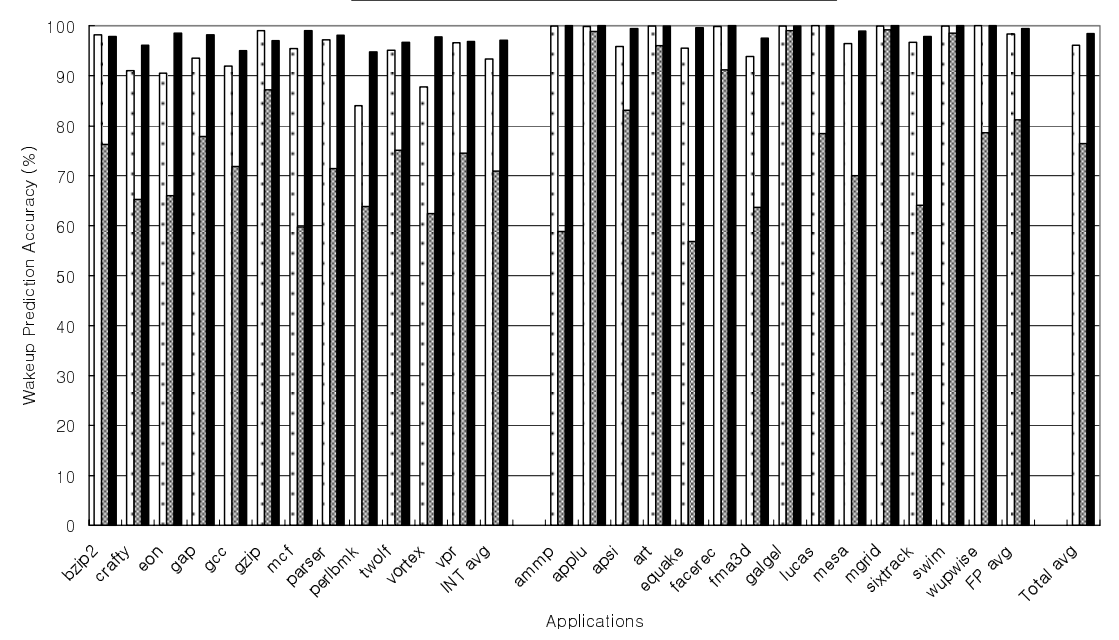

Figure 9. Wakeup prediction accuracy per fetch, Including bitline precharging accuracy (direct-mapped)

Figure 9 shows the wakeup prediction accuracy (\#fetches with a target-mispredicted branch/\# fetches) in the direct-mapped cache. For noaccess-JITA/NTSBP, the wakeup prediction accuracy includes bitline precharging prediction accuracy. Average wakeup prediction accuracy of noaccess-JITA/NTSBP is as high as 96.1\%, although for perlbmk, the prediction accuracy is only $84.0 \%$. On the other hand, the average wakeup prediction accuracy of the DHS-Bank-PA is $76.5 \%$. In the proposed on-demand policy, average wakeup prediction accuracy is as high as $98.4 \%$. Moreover even in the worst case, the wakeup prediction accuracy is no worse than $94 \%$.

Though the accuracy of noaccess-JITA/NTSBP is harmed more by bitline precharging prediction than other techniques, the super-drowsy cache with bitline gating noaccess-JITA/NTSBP reduces the leakage-related energy much more than the drowsy cache with noaccess-JITA without bitline gating in all applications [9]. 


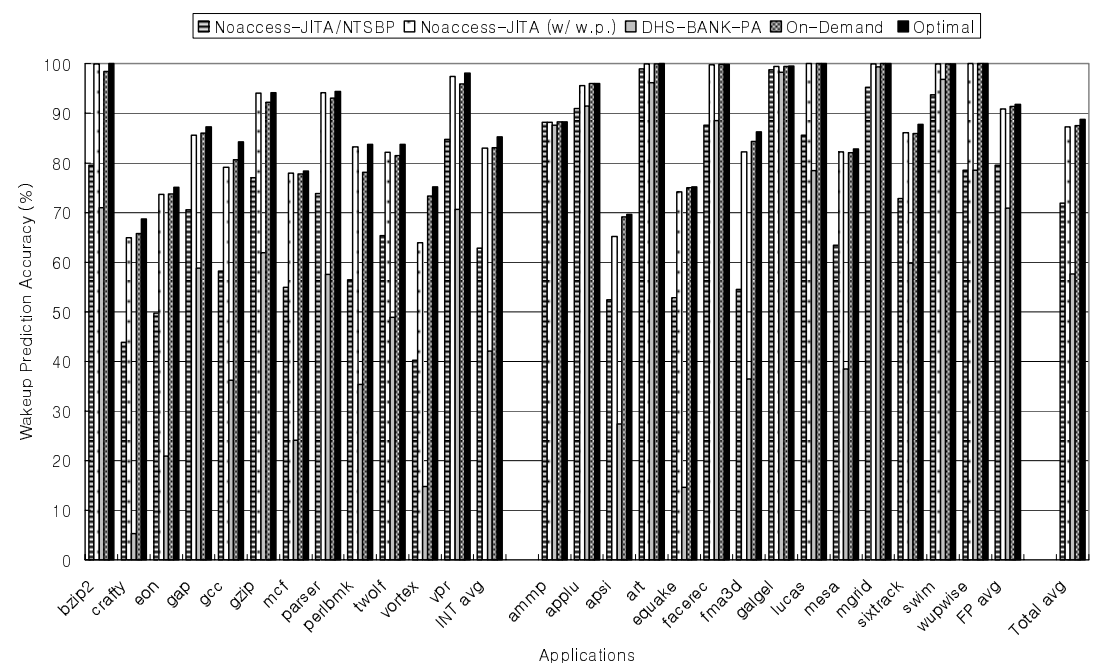

Figure 10. Wakeup prediction accuracy per fetch, Including bitline precharging and way prediction accuracy (4-way set-associative)

Figure 10 shows the wakeup prediction accuracy, including bitline precharging and way prediction accuracy in the 4-way set-associative cache. The accuracy of the optimal policy implies the way prediction accuracy. Please note that the results are not per instruction but per fetch. Average accuracy of the noaccess-JITA/NTSBP is $71.9 \%$ since a set-associative cache make it more difficult to predict sub-bank precharging. However, the noaccess-JITA (utilizing w.p.) and the proposed on-demand policy shows $87.3 \%$ and $87.6 \%$ accuracy, respectively which is close to the accuracy (way prediction accuracy) of the optimal policy. The accuracy of DHS-Bank-PA is as low as $57.6 \%$, on average, which might result in severe performance degradation. This is caused by flushing the previous sub-bank when execution jumps from one sub-bank to another, since the sub-bank hoppings are much more frequent in a setassociative cache.

\subsection{Execution Time}

Even one percent increase of execution time leads to substantial increase of the total processor energy, which might counterbalance the reduced L1 instruction cache leakage. Thus, it is crucial to maintain execution time close to the base model. We only show the proposed policy of Figure 2, since there is negligible difference from that of Figure 3.

When a wakeup misprediction (including precharging misprediction and way misprediction) and an instruction cache miss occur at the same time, the wakeup penalty is hidden by the cache miss penalty. Thus, the wakeup prediction accuracy is related to the execution time but this is not always exactly proportional.

Figure 11 shows the execution time normalized to the base model in the direct-mapped cache. The noaccessJITA/NTSBP increases execution time by $0.65 \%$, on average but recall that leakage reduction is only $67.5 \%$. In some applications, the increase of the execution time is more than 3\%. The DHS-Bank-PA shows $2.7 \%$ execution time increase, on average. Even worse, in some applications, execution time is increased by more than $12 \%$. The proposed on-demand policy increases the execution time by only $0.26 \%$ and $2.3 \%$, on average and in worst case, respectively.

Figure 12 shows the execution time normalized to the base model in the 4-way set-associative cache. The increases of execution time are $2.09 \%, 0.15 \%, 5.36 \%$, and $0.27 \%$ for noaccess-JITA/NTSBP, noaccess-JITA (utilizing w.p.), DHS-Bank-PA, and the proposed on-demand policy. Though the noaccess-JITA/NTSBP increases the execution time by inaccurate next sub-bank prediction, the noaccess-JITA (utilizing w.p.) does not since it utilizes the 2-read port way predictor which is more accurate than the NTSBP. In equake, The DHS-Bank-PA degrades the performance as much as $30.1 \%$, which is too severe to be tolerated. 


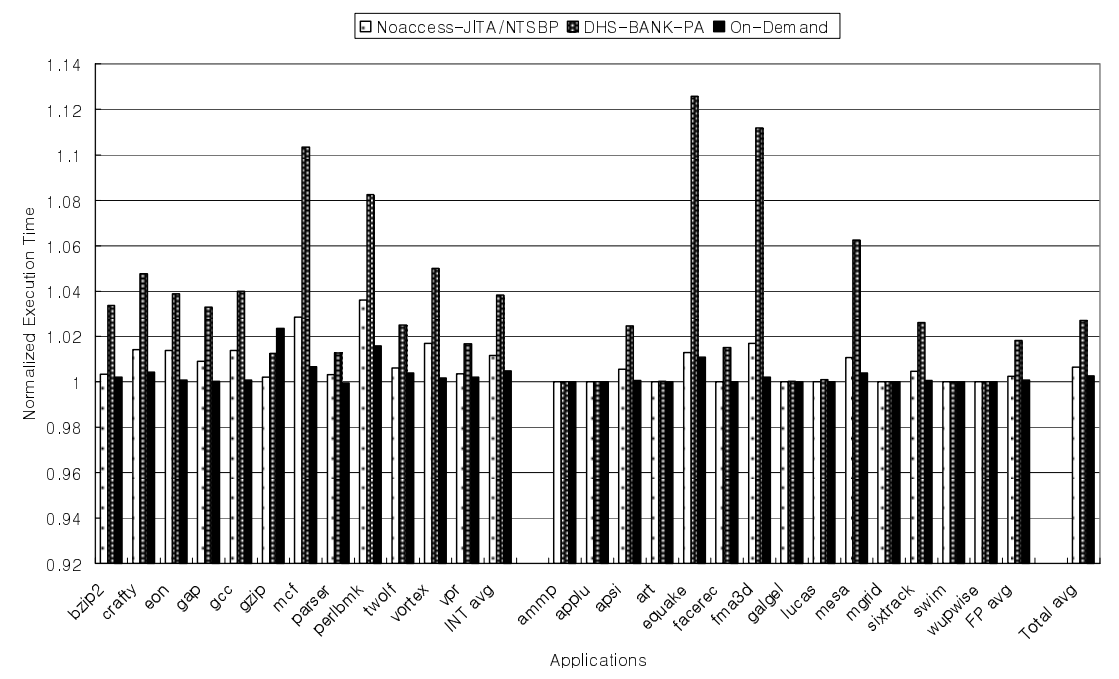

Figure 11. Normalized execution time (direct-mapped)

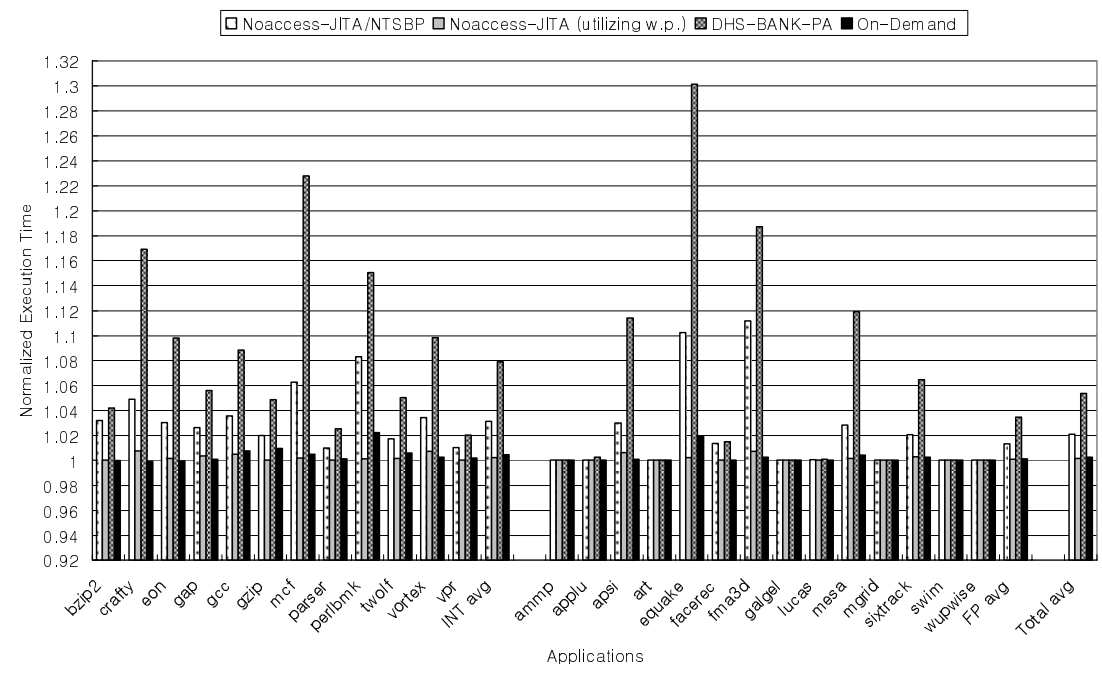

Figure 12. Normalized execution time (4-way set-associative)

\subsection{Total processor energy and $\mathrm{ED}^{2}$}

Figure 13 shows average total processor energy and average $\mathrm{ED}^{2}\left(\right.$ Energy*Delay $\left.^{2}\right)$ for all SPEC2000 applications in the direct-mapped cache (Please note that the "on-demand" graphs are almost overlapped with the "optimal" graph in Figure 13 and Figure 14). Since the proposed policy and the optimal policy show similar performance and leakage-related energy reduction as shown in the previous sub-sections, the total energy is also similar, regardless of leakage-related energy ratio. The noaccess-JITA/NTSBP reduces the total processor energy more than the DHSBank-PA in the leftmost bars, since the performance degradation by the DHS-Bank-PA is significant. However, as the leakage-related energy in the instruction cache accounts for more of the total processor energy, total energy of the DHS-Bank-PA becomes less than that of the noaccess-JITA/NTSBP. For ED ${ }^{2}$, there is negligible difference between the proposed policies and the optimal policy

Figure 14 shows average total processor energy and average $\mathrm{ED}^{2}\left(\right.$ Energy*Delay $\left.^{2}\right)$ for all SPEC2000 applications in the 4-way set-associative cache. When the leakage-related energy accounts for small portion of total processor energy, the noaccess-JITA (utilizing w.p.) is the only competitor of the proposed on-demand policy, as shown in Figure 14. However, as the leakage-related energy portion increases, the gap between the noaccess-JITA (utilizing w.p.) and the on-demand policy gets larger in terms of total processor energy and $\mathrm{ED}^{2}$, both. Moreover, there is little 
difference between the on-demand policies and the optimal policy.

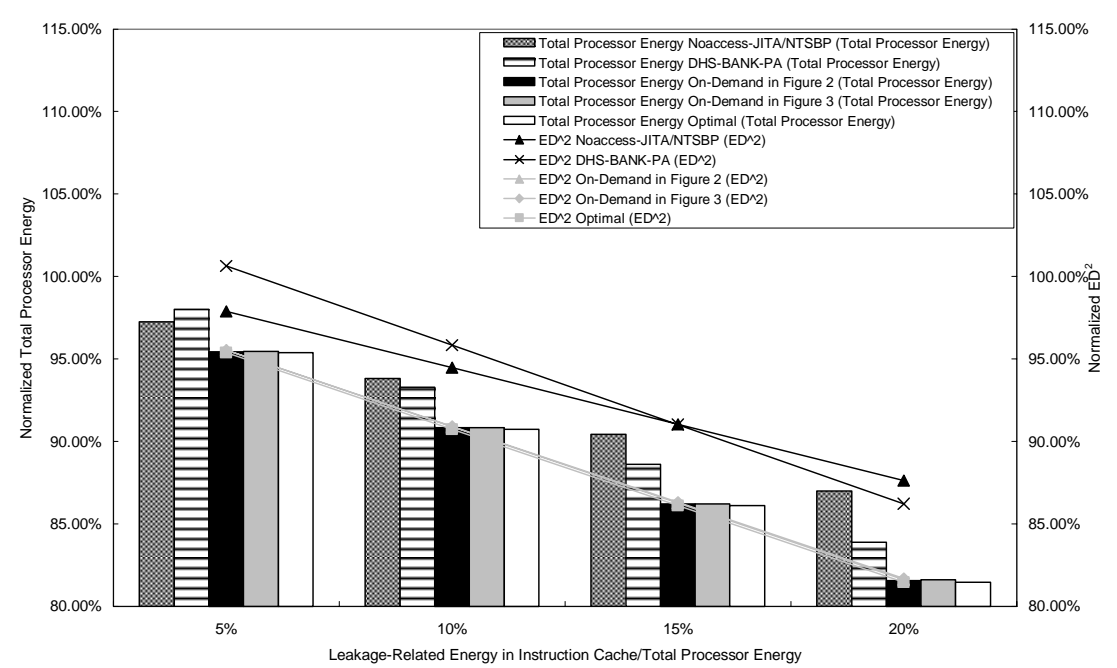

Figure 13. Total processor energy and $\mathrm{ED}^{2}$ (direct-mapped)

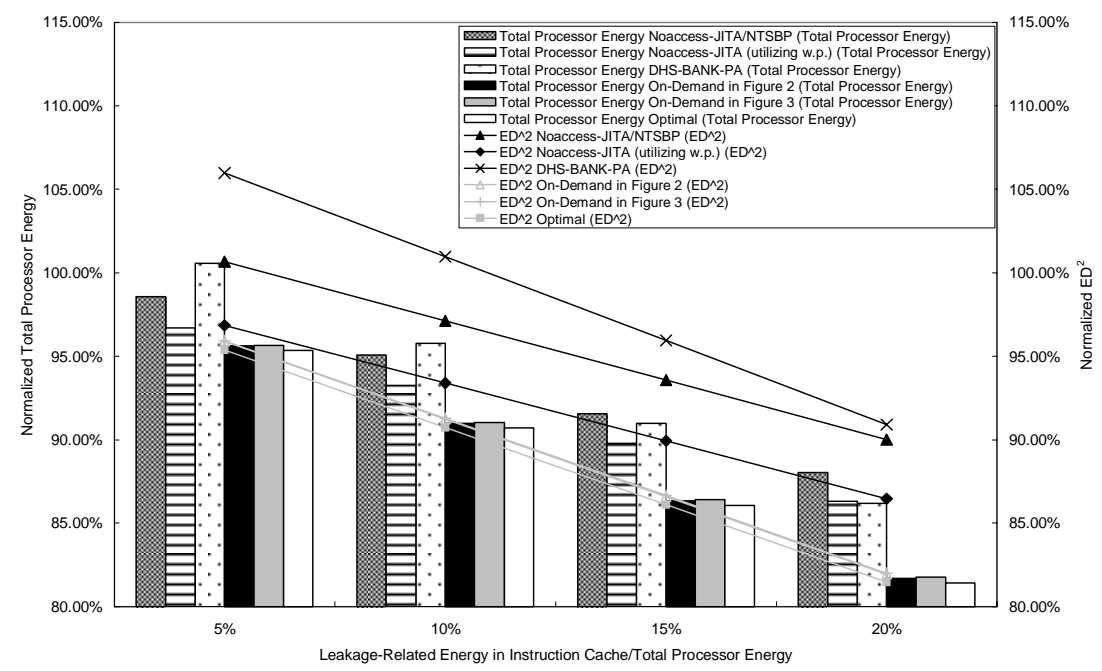

Figure 14. Total processor energy and $\mathrm{ED}^{2}$ (4-way set-associative)

\subsection{Combining with Other Leakage Saving Circuit Techniques}

The proposed policy can be adopted for other leakage saving circuit techniques, as long as the wakeup penalty is one cycle. Though other techniques, such as ABB MTCMOS [17] and DRG [1], have longer wakeup penalty in current and next generation process technologies, the development of process technology and new circuit technology might be able to reduce the wakeup penalty in the future. In Figure 15, we normalized average instruction cache leakage energy to the base model for SPEC2000 applications. We present only one graph in Figure 15, since both results (direct-mapped cache and 4-way set-associative cache) are almost overlapped (within $0.01 \%$ ). In this graph, we excluded the turn-on energy which is negligible to the total instruction cache leakage energy. Figure 15 makes it possible to estimate how much leakage energy is reduced, when other leakage saving circuit techniques are applied, as long as the wakeup penalty is one cycle. The execution time increased by the wakeup penalty is same as shown in Figure 11 and Figure 12. 


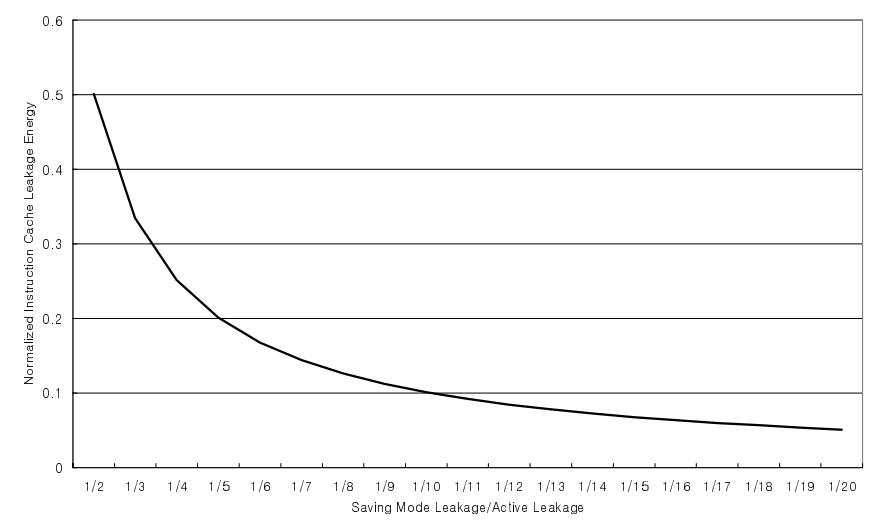

Figure 15. Instruction cache leakage reduction combined with other leakage saving circuit techniques

\subsection{Comparison of Hardware Overhead}

For a wakeup prediction policy, hardware overhead is inevitable in additional to the DVS control circuitry. We compare the hardware overhead of each policy. In the noaccess-JITA/NTSBP, one bit per cache line is required in order to detect whether the cache line is accessed or not in the fixed time period. In addition, the NTSBP has $1 \mathrm{~K}$ entries (3 bits/entry). The noaccess-JITA (utilizing w.p.) requires one bit per cache line same as the noaccess-JITA. In addition, it needs 2-read port way predictor for bitline precharging (sub-bank) prediction. In the DHS-Bank-PA, one bit per cache line is also required to store the access history. Additionally, ten bits (half for the target basic block counter and the other half for the fall-through basic block counter) are required to locate a hotspot [5]. Since the BTB has 1024 entries, the total storage overhead is 10K. For the proposed policy, only a small register (ex. 10 bit for our 1024-entry cache) is needed to record the most recently accessed cache line. Table 5 presents the total hardware overhead for each policy. The hardware overhead is crucial, since it not only increases chip area but also incurs extra dynamic/leakage energy.

\begin{tabular}{|c|c|c|c|}
\hline Noaccess-JITA/NTBSP & $\begin{array}{l}\text { Noaccess-JITA } \\
\text { (utilizing w.p.) }\end{array}$ & DHS-Bank-PA & On-Demand \\
\hline $\begin{array}{c}4 \mathrm{~K} \text { bit } \\
(=1024+1024 * 3)\end{array}$ & $\begin{array}{c}\text { 1K bit + 2-read port } \\
\text { way predictor (instead } \\
\text { of 1-read port way } \\
\text { predictor) }\end{array}$ & $\begin{array}{c}11 \mathrm{~K} \text { bit } \\
(=1024+1024 * 10)\end{array}$ & $\begin{array}{c}10 \text { bit } \\
\left(=\log _{2} 1024\right)\end{array}$ \\
\hline
\end{tabular}

\section{Table 5. Hardware overhead for the policies}

(Please remind that the storage size is directly related to leakage energy, though we do not consider the dynamic/leakage energy from this hardware overhead in the evaluation)

\section{Conclusions and Future Work}

In this paper, we propose an on-demand wakeup prediction policy using the branch prediction information. Our goal is not only less energy consumption but also consistent near-optimal performance. The noaccess-JITA/NTSBP and the noaccess-JITA (w/ w.p.) show competitive performance consistently but their energy consumption is more than four times of the proposed policy, on average. The DHS-Bank-PA reduces leakage-related energy significantly but it increases the execution time by more than $10 \%$ in many cases. In several cases, the increase is more than $20 \%$. The proposed policy degrades the performance by only $0.26 \sim 0.27 \%$, on average, and $1.6 \sim 1.9 \%$ for the worst case. At the same time, leakage energy is almost eliminated since only one (or two) cache line is active while all other lines are in the drowsy mode. This is especially beneficial for controlling leakage in future instruction caches which might be much larger. The leakage energy reduction by the proposed policy is on average 92.2 92.5\%, almost identical to the reduction by the optimal policy $(92.6 \%)$. The total processor energy and the $\mathrm{ED}^{2}$ of the proposed policy are also almost identical to those of the optimal policy. Therefore, we conclude that the proposed on-demand wakeup prediction policy is near-optimal.

We believe that there is no reason to try to reduce remaining leakage by adopting non-state-preserving techniques, at the risk of severe performance degradation. The proposed policy can be adopted for other state-preserving leakage saving circuit techniques as long as the wakeup penalty is at most one cycle.

In this paper, we apply the on-demand wakeup policy to the tag part of the instruction cache. However, when the 
tag part is always in the active mode, the tag access can be moved to the wakeup stage, leading to tag matching in the wakeup stage (or one cycle before the fetch stage). This results in 100\% accurate way prediction without any way predictor. The trade-off between the reduction of leakage energy in the tag part and perfect way prediction will be an interesting research topic. We also plan to apply the proposed policy to low-end embedded processors, where instruction fetch/issue rate is lower and the instruction cache may comprise a much larger fraction of chip area, resulting in more total processor energy reduction compared to high-end processors.

\section{Acknowledgements}

This work was funded in part by the National Science Foundation under grant nos. CAREER CCR-0133634, CCF0429765, the Army Research Office under grant no. W911NF-04-1-0288, a Faculty Partnership Award from IBM T.J. Watson, a gift from Intel MTL, an Excellence Award from the Univ. of Virginia Fund for Excellence in Science and Technology, and the IT National Scholarship Program from IITA \& MIC, Korea.

\section{References}

[1] A. Agarwal, L. Hai, and K. Roy. A Single-Vt Low-Leakage Gated-Ground Cache for Deep Submicron. IEEE Journal of Solid-State Circuits. Vol. 38, Feb, 2003, pp. 319-328.

[2] T. Austin, E. Larson, and D. Ernst. Simplescalar: An Infrastructure for Computer System Modeling. IEEE Computer Magazine. vol. 35, 2002, pp. 59-67.

[3] K. Flautner, N. S. Kim, S. Martin, D. Blaauw, T. Mudge. Drowsy Caches : Simple Techniques for Reducing Leakage Power. Proc. of Int. Symp. on Computer Architecture, 2002, pp. 148-157.

[4] F. Hamzaoglu, Y. Ye, A. Keshavarzi, K. Zhang, S. Narendra, S. Borkar, M. Stan, and V. De. Analysis of Dual-VT SRAM cells with Full-Swing Single-Ended Bit Line Sensing for On-Chip Cache. IEEE Transaction on VLSI Systems, vol. 10, April 2002, pp. 91-95.

[5] J. S. Hu, A. Nadgir, N. Vijaykrishnan, M. J. Irwin, M. Kandemir. Exploiting Program Hotspots and Code Sequentiality for Instruction Caches Leakage Management. Proc. of Int. Symp. on Low Power Electronics and Design, 2003, pp. 593-601.

[6] S. Kaxiras, Z. Hu, and M. Martonosi. Cache decay: Exploiting generational behavior to reduce cache leakage power. Proc. of Int. Symp. on Computer Architecture, 2001, pp 240-251.

[7] R. Kessler. The Alpha 21264 Microprocessor. IEEE Micro Magazine. 1999, pp.24-36.

[8] N. S. Kim, K Flautner, D. Blaauw, and T. Mudge. Circuit and Microarchitectural Techniques for Reducing Cache Leakage Power. IEEE Transaction on VLSI Systems, vol.12, no. 2, Feb. 2004, pp 167-184.

[9] N. S. Kim, K. Flautner, D. Blaauw, T. Mudge. Single-Vdd and Single-Vt Super-Drowsy Techniques for LowLeakage High-Performance Instruction Caches, Proc. of Int. Symp. on Low Power Electronics and Design, 2004, pp.54-57.

[10] L. Li, V. Degalahal, N. Vojaykrishnan, M. Kandemir, and M. J. Irwin. Soft Error and Energy Consumption Interactions: A Data Cache Perspective. Proc. of Int. Symp. on Low Power Electronics and Design, 2004, pp. 132137.

[11] L. Li, I. Kadayif, Y-F. Tsai, N. Vijaykrishnan, M. Kandemir, M. J. Irwin and A. Sivasubramaniam. Leakage Energy Management in Cache Hierarchies. Proc. of Int. Conf. on Parallel Architectures and Compilation Techniques, 2002, pp.131-140.

[12] Y. Li, D. Parikh, Y. Zhang, K. Sankaranarayanan, M. Stan, and K. Skadron. State-Preserving vs. Non-StatePreserving Leakage Control in Caches. Proc. of the Design Automation and Test in Europe Conference. 2004, pp. 22-27.

[13] S. Manne, A. Klauser, and D. Grunwald, Pipeline Gating : Speculation Control for Energy Reduction. Proc. of Int. Symp. on Computer Architecture, 1998, pp.132-141.

[14] S. McFaring. Combining Branch Predictors. Technical Note TN-36. DEC June 1993.

[15] Y. Meng, T. Sherwood and R. Kastner. On the Limits of Leakage Power Reduction in Caches. Proc. of Int. Symp. on High-Performance Computer Architecture. 2005.

[16] M. Milenkovic, A. Milenkovic, and J. Kulick. Demystifying Intel Branch Predictor. Proc. of Workshop on Duplicating, Deconstucting and Debunking (in conjunction with ISCA-29). 2002.

[17] K. Nii et. al. A Low Power SRAM Using Auto-Backgate-Controlled MT-CMOS. Proc. of Int. Symp. on Low Power Electronics and Design, 1998, pp. 293-298.

[18] M. Powell, S.-H. Yang, B. Falsafi, K. Roy, and T. N. Vijaykumar. Gated-Vdd : A circuit technique to reduce leakage in deep-submicron cache memories. Proc. of Int. Symp. on Low Power Electronics and Design, 2000, pp 90-95.

[19] G. Reinman and B. Calder. Using a Serial Cache for Energy Efficient Instruction Fetching. Journal of Systems 
Architecture. vol. 50 , issue 11, 2004, pp.675-685.

[20] S. Yang and B. Falsafi. Near-Optimal Precharging in High-Performance Nanoscale CMOS Caches. Proc. of Int. Symp. on Microarchitecture, 2003.

[21] S. Yang, M. Powell, B. Falsafi, K. Roy, and T. Vijaykumar. An Integrated Circuit/Architecture Approach to Reducing Leakage in Deep-Submicron High-Performance I-Caches. Proc. of Int. Symp. on High-Performance Computer Architecture, 2001, pp.147-157.

[22] W. Zhang, J. Hu, V. Degalahal, M. Kandemir, N. Vijaykrishnan, and M. J. Irwin. Compiler-Directed Instruction Cache Leakage Optimization. Proc. of Int. Symp. on Microarchitecture, 2002, pp.208-218.

[23] ARM. ARM 1136 Technical Reference Manual. Available in http://www.arm.com

[24] ITRS (International Technology Roadmap for Semiconductor). Available in http://public.itrs.net.

[25] Standard Performance Evaluation Corp.. Available in http://www.specbench.org.

[26] VAR Business, Intel Clears up Post-Tejas Confusion, Available in http://www.varbusiness.com/sections /news/breakingnews.jhtml?articleId=18842588 